\title{
Enterprise stent-within-stent embolization of a basilar artery perforator aneurysm
}

\author{
Eric M. Deshaies ${ }^{1}$, Walter Jacobsen ${ }^{2}$, Satish Krishnamurthy ${ }^{3}$ \\ ${ }^{1}$ Director of the SUNY Upstate Neurovascular Center; Director of Cerebrovascular, Endovascular, and Skull Base Neurosurgery. \\ Assistant Professor of Neurosurgery, Neuroscience and Physiology; SUNY Upstate Medical University, Syracuse NY; \\ ${ }^{2}$ Department of Neurosurgery, SUNY Upstate Medical University, Syracuse NY; \\ ${ }^{3}$ MCh; Professor of Neurosurgery; Director of Minimally Invasive Neurosurgery, Department of Neurosurgery, SUNY Upstate Medi- \\ cal University, Syracuse NY. \\ Email: deshaiee@upstate.edu; krishnsa@upstate.edu
}

Received 11 August 2011; revised 22 September 2011; accepted 15 October 2011.

\section{ABSTRACT}

Objective and Importance-Vertebrobasilar artery aneurysms can be very challenging to treat and are preferentially dealt with using endovascular techniques, since they are associated with lower risk than surgical clipping. Small aneurysms located on perforating arteries can pose problems though, as their dimensions may not be favorable for coiling and leave the endovascular surgeon without many options. We present a basilar perforating artery aneurysm that was successfully embolized using a stent-within-stent technique. Clinical Presentation-A 47 year old female presented with a Hunt-Hess 3, Fisher Grade-3 subarachnoid hemorrhage with blood in the pre-pontine cistern. Initial imaging (CT angiogram and digital subtraction angiography) did not reveal an aneurysm. Follow-up angiography on post-bleed day eight demonstrated a three-millimeter basilar perforating artery aneurysm. After an unsuccessful coiling attempt a closed-cell stent-within-stent technique was used to divert flow away from the aneurysm neck to induce aneurysm thrombosis. Intervention (or Technique)-Multiple attempts were made to access and stabilize a microcatheter in the small basilar perforator artery aneurysm in order to deliver coils for endovascular embolization; this could not be done safely. Therefore a closed-cell $4.5 \times 22 \mathrm{~mm}$ Enterprise stent (Cordis Neurovascular, Inc., Miami Lakes, Florida) was deployed in the basilar artery across the origin of the perforator aneurysm in order for the stent tines to divert flow away from the aneurysm neck and induce thrombosis. Persistant brisk flow within the aneurysm continued however, and a second closedcell $4.5 \times 22 \mathrm{~mm}$ Enterprise stent was placed within the

*Dr. Eric M. Deshaies is a Physician Consultant to eV3, Microvention, and Integra Corporations. first one to increase the stent metal surface area across the aneurysm neck to further reduce flow into the aneurysm. Subsequently, angiography demonstrated stagnant blood flow in the aneurysm dome and the aneurysm spontaneously thrombosed, sparing all associated vessels. Conclusion-Stent-within-stent technique should be considered as a possible endovascular treatment option for small side-wall and perforator artery aneurysms that can't be safely treated with coils or embolic agents.

Keywords: Aneurysm; Basilar, Enterprise; Stent; Subarachnoid Hemorrhage

\section{OBJECTIVE AND IMPORTANCE}

Aneurymal subarachnoid hemorrhage affects 11 patients per 100,000 population each year (27,000 aneurysm ruptures in the United States alone, annually) [1,2]. Aneurysms can form on any intracranial artery, but most commonly occur in the Circle of Willis. Most of the aneurysms of the vertebrobasilar system are found at the basilar artery bifurcation, but can also occur at the origin of other arteries at their bifurcation points [3]. One example is an aneurysm arising from the origins of the basilar perforating arteries. Aneurysms in the vertebrobasilar territory are preferably treated with endovascular coiling because the risks of treatment are lower than surgical clip ligation $[4,5]$.

Aneurysms arising from the sides of arteries, called sidewalled aneurysms, can pose a particular challenge to treat with endovascular techniques because the microcatheter may not naturally take the conformation needed to access the aneurysm so that coils can be delivered into the lesion. When confronted with a ruptured side-walled aneurysm that can't be accessed and secured with coil embolization, other endovascular options are not typically 
available, meaning that the aneurysm can't be secured without putting the patient through the high risks of surgical intervention near the brainstem.

Here, we present the successful treatment of a ruptured three-millimeter side-walled basilar perforating artery aneurysm that could not be secured with traditional endovascular means. Given that the location was not optimal for surgical intervention, and endovascular treatment was thought to be the better option, the author (EMD) performed a novel endovascular intervention to successfully divert blood flow away from the aneurysm neck and occlude the aneurysm, using a stent-within-stent technique.

\section{CLINICAL PRESENTATION}

A 47-year old female presented with a Hunt-Hess 3, Fisher Grade-3 subarachnoid hemorrhage (SAH) in the pre-pontine cistern. Initial CT angiogram and digital subtraction angiography did not reveal an aneurysm or vascular malformation. On the follow-up angiogram eight days later, a three-millimeter basilar perforating artery aneurysm was identified (Figure 1). Due to the high morbidity and mortality associated with surgical intervention, endovascular intervention was deemed safer and the patient was brought to the endovascular suite for embolization of the aneurysm.

\section{INTERVENTION}

Coil embolization was attempted numerous times but could not be performed successfully secondary microcatheter instability within the aneurysm neck. Each of the six times the aneurysm was catheterized and coil insertion was attempted, the microcatheter displaced from the aneurysm. At this point, coil embolization was abandoned as a potentially safe treatment option for this aneurysm because multiple attempts increase the risk of aneurysm

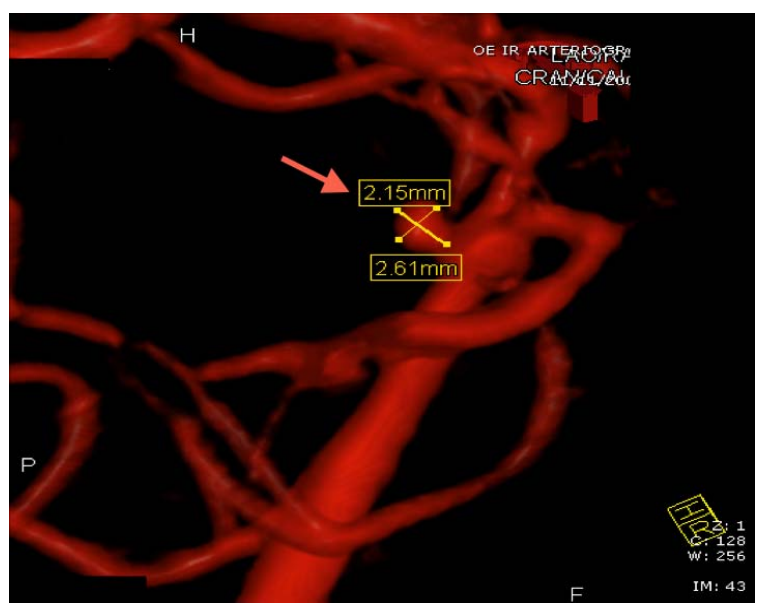

Figure 1. 3D digital subtraction cerebral angiogram (mid-arterial phase) of the basilar artery demonstrating a $<3 \mathrm{~mm}$ dorsal basilar perforator artery aneurysm (arrow). re-rupture from difficult access. At that point, a $4.5 \times 22$ mm closed-cell Cordis Enterprise stent was deployed in the basilar artery across the perforating aneurysm origin. A closed cell stent configuration was chosen by the endovascular neurosurgery (EMD) because it would potentially provide better surface coverage of the aneurysm neck, with the intent that it would divert blood flow from the aneurysm neck and induce thrombosis. Additionally, there was concern of a theoretical risk that the stent tines of an open cell stent could bend into the aneurysm and cause a perforation and re-rupture. After placement of the stent, angiography still showed persistent brisk flow within the aneurysm, so a second stent was placed inside the first one to further reduce the total surface area of the inflow zone of the aneurysm neck. After both Cordis Enterprise stents were deployed across the aneurysm origin, angiography demonstrated that the blood flow into the aneurysm was diminished and the outflow was signifycantly delayed (Figure 2(a) and (b)).

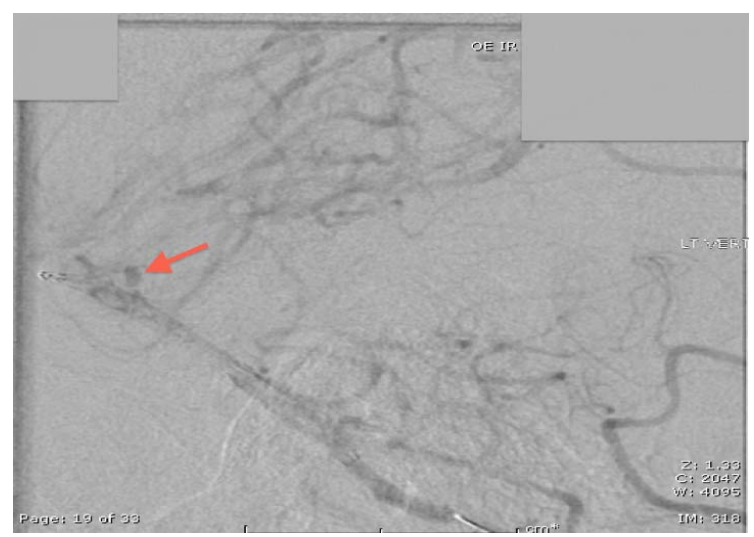

(a)

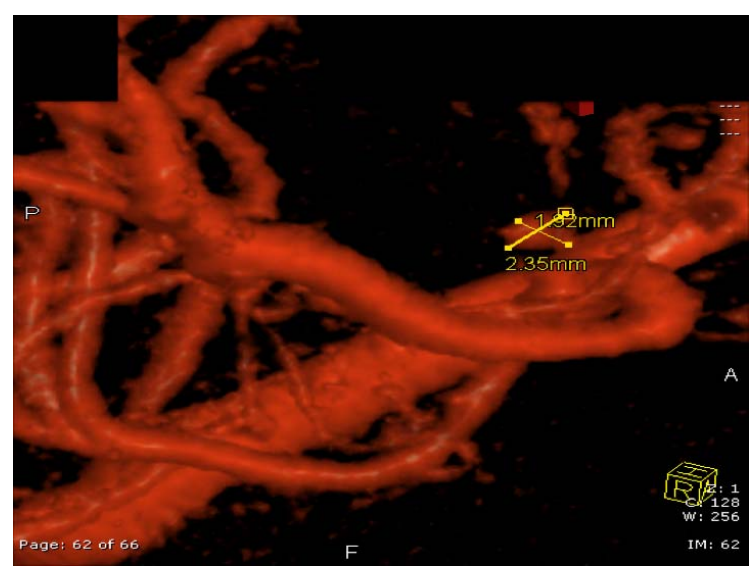

(b)

Figure 2. (a) 2D digital subtraction cerebral angiogram of the basilar artery after completing the Enterprise stent-within-stent technique. There is reduced flow into the aneurysm and delayed emptying of the contrast in the late arterial phase. (b) 3D digital subtraction angiogram demonstrating the residual aneurysm (arrow). 
The patient was placed on aspirin therapy alone to simultaneously lower the risk of in-stent thrombosis yet allow the aneurysm to thrombose, and also allow for potentially needed external ventricular drain placement in the future if needed. Repeated cerebral angiography the next day demonstrated that the aneurysm dome was almost completely thrombosed. At this point she was placed on an intravenous drip of abciximab (ReoPro, Centocor, Philadelphia, PA), a short-acting glycoprotein IIb/IIIa receptor antagonist, to minimize the risk of in-stent thrombosis. A second angiogram on day three showed that the aneurysm was filling again, likely secondary to the abciximab. Hence abciximab was stopped again and she was continued on aspirin alone until it was decided that a ventriculosotomy was not needed, after which time clopidogrel (Plavix 75 mg oral, daily) was added to her antiplatelet regimen. The patient recovered well and CT angiogram performed prior to discharge showed no residual aneurysm dome filling. The patient was discharged home on post-bleed day 20 with a standard antiplatelet regimen of aspirin (325 mg oral, daily) and clopidogrel (75 mg oral, daily) therapy for 12 weeks. Six month follow-up digital subtraction angiography showed that the aneurysm had thrombosed and the stents remained patent without any evidence of stent migration or stenosis (Figure 3).

\section{CONCLUSIONS}

Ruptured basilar perforating artery aneurysms can pose challenges both in diagnosis and treatment. We could find three articles that reported four patients with similar aneurysms [6-8]. All five patients (including the present

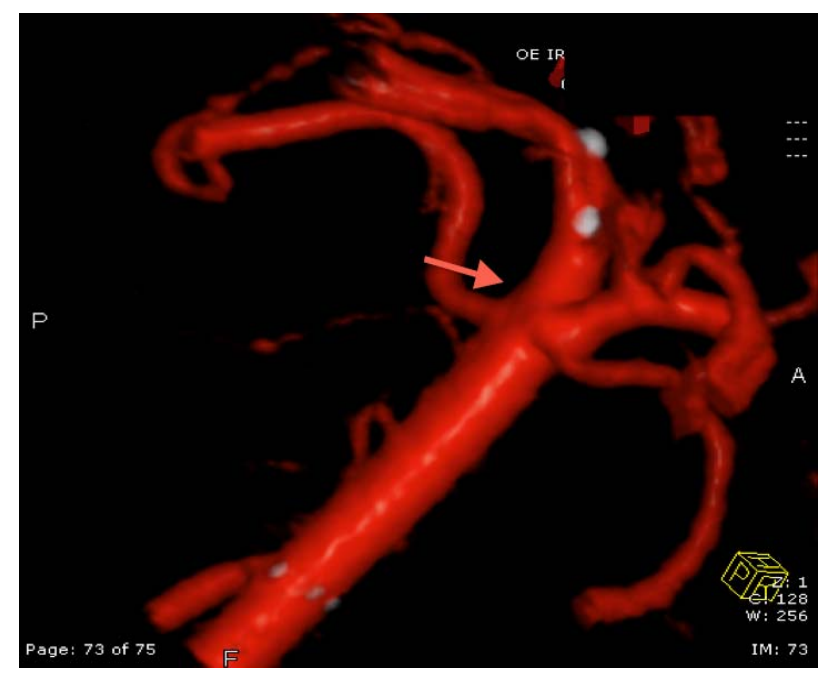

Figure 3. Six month follow-up 3D digital subtraction cerebral angiogram (mid-arterial phase) of the basilar artery demonstrating complete occlusion of the basilar perforator artery aneurysm (arrow), no in-stent stenosis, and no stent migration (gray). one reported here) had no aneurysm found on the initial angiogram and the aneurysms were demonstrated on a subsequent study. A perimesencephalic location of the SAH, particularly in conjunction with an angiographically negative study, may lull the treating neurosurgeon into thinking that these patients have non-aneurysmal SAH. Furthermore, all of the reported cases treated prior to our report, were treated surgically as endovascular coiling was not possible. Flow diverting stents, such as the Pipeline Embolization Device (eV3, Irvine, California) and the Silk Stent (Balt, Montmorency, France), have been shown to be unsafe for use in the basilar artery since they are associated with a high risk of basilar perforating artery occlusion and brainstem stroke.

Traditional intracranial arterial stents can be divided into “open” and “closed” cell designs. The “open” cell design has holes in the stent wall that do not have metal around the entire circumference of the cells, whereas the "closed" cell stent has metal encircling the entire cell circumference (Figure 4(a) and (b)). The Cordis Enterprise stent is a closed-cell nitinol stent, with each cell diameter measuring less than 1.3 French. We had postulated that placing the closed-cell stent across the aneurysm neck would divert flow away from the aneurysm neck and impede outflow of blood from the aneurysm, both promoting thrombosis [9]. An additional theoretical advantage of using the closed-cell design is that is would lessen the risk of the stent tines perforating the aneurysm. A second stent was placed within the first to further reduce the total stent cell size and hence, the cross-sectional area of the aneurysm inflow zone.

In vitro and in vivo studies on aneurysm hemodynamics after stent placement have consistently demonstrated reduced flow and shear-stress inside the aneurysm after stent placement [9-15]. Stents also reduce the pressure in the distal zone of the aneurysm neck and dome [10]. All of these hemodynamic changes are favorable for promoting intra-aneurysmal flow stasis and thrombosis. The

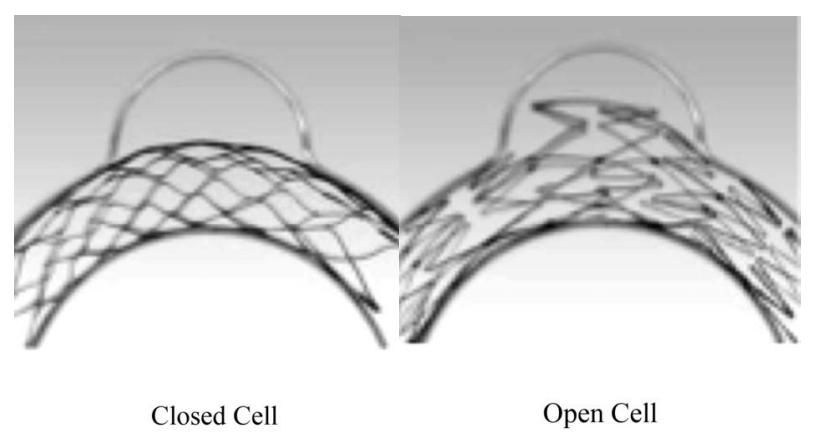

(a)

(b)

Figure 4. (a) Enterprise (Cordis, Corporation, Johnson and Johnson) stent with closed-cell technology. (b) Neuroform microdelivery stent (Boston Scientific) stent with open-cell technology. 
stent surface provides scaffolding for neo-intimal proliferation and healing of the aneurysm neck, which can further reduce flow into the aneurysm once the stent "heals" into place.

In conclusion, stent-within-stent placement across the neck of a side-walled aneurysm should be considered an alternative treatment for aneurysms that cannot be treated with coil embolization, flow-diverting stents, or embolic glue agents. This technique may also apply to endovascular treatment of small blister aneurysms in other arterial locations that are difficult to coil within the Circle of Willis. Acute in-stent thrombosis from the metal-to-blood interface and delayed in-stent stenosis from neointimal hyperplasia, are real risks that must be monitored closely.

\section{REFERENCES}

[1] Schievink, W.I. (1997) Intracranial aneurysms. New England Journal of Medicine, 336, 28-40. doi:10.1056/NEJM199701023360106

[2] Wijdicks, E.F., Kallmes, D.F., Manno, E.M., Fulgham, J.R. and Piepgras, D.G. (2005) Subarachnoid hemorrhage: Neurointensive care and aneurysm repair. Mayo Clinic Proceedings, 80, 550-559. doi:10.4065/80.4.550

[3] Rhoton, A.J. (2002) Aneurysms. Neurosurgery, 51, 121158. doi:10.1097/00006123-200210001-00004

[4] Leusseveld, E.B.E., Nijssen, P.C., Van Rooij, W.J., Sluzewski, M., Tulleken, C.A., Wijnalda, D., Schellens, R.L., Van der Graaf, Y. and Rinkel, G.J. (2002) Endovascular coiling versus neurosurgical clipping in patients with a ruptured basilar tip aneurysm. Journal of Neurology Neurosurgery and Psychiatry, 73, 591-593. doi:10.1136/jnnp.73.5.591

[5] Van der Schaaf I, A.A., Wermer, M., Molyneux, A., Clarke, M., Van Gijn, J. and Rinkel, G. (2005) Endovascular coiling versus neurosurgical clipping for patients with aneurysmal subarachnoid hemorrhage. Cochrane Database of Systematic Reviews, October 19.
[6] Ghogawala Z, S.J. and Ogilvy, C.S. (1996) Distal basilar perforator artery aneurysm: Case report. Neurosurgery, 39, 393-396. doi:10.1097/00006123-199608000-00034

[7] Mathieson CS, B.P., Jenkins, S. and Hanzely, Z. (2010) An unusual case of spontaneous subarachnoid hemorrhage - a ruptured aneurysm of a basilar perforator artery. British Journal of Neurosurgery, 24, 291-293. doi:10.3109/02688690903572095

[8] Sanchez-Mejia RO, L.M. (2007) Distal aneurysms of basilar perforating and circumferential arteries. Report of three cases. Journal of Neurosurgery, 107, 654-659. doi:10.3171/JNS-07/09/0654

[9] Wanke, I. and Forsting, M. (2008) Stents for intracranial wide-necked aneurysms: More than mechanical protection. Neuroradiology, 50, 991-998. doi:10.1007/s00234-008-0460-0

[10] Aenis, M., et al. (1997) Modeling of flow in a straight stented and nonstented side wall aneurysm model. Journal of Biomechanical Engineering, 119, 206-212. doi:10.1115/1.2796081

[11] Geremia, G., Haklin, M. and Brennecke, L. (1994) Embolization of experimentally created aneurysms with intravascular stent devices. American Journal of Neuroradiology, 15, 1223-1231.

[12] Henkes, H., et al. (2004) Coil treatment of a fusiform upper basilar trunk aneurysm with a combination of "kissing” neuroform stents, TriSpan-, 3D- and fibered coils, and permanent implantation of the micro-guidewires. Neuroradiology, 46, 464-468.

[13] Liou, T.M., Liou, S.N. and Chu, K.L. (2004) Intra-aneurysmal flow with helix and mesh stent placement across side-wall aneurysm pore of a straight parent vessel. Journal of Biomechanical Engineering, 126, 36-43. doi:10.1115/1.1644566

[14] Sadasivan, C., et al. (2002) Angiographic quantification of contrast medium washout from cerebral aneurysms after stent placement. American Journal of Neuroradiology, 23, 1214-1221.

[15] Wanke, I. (2008) Stents for intracranial aneurysms: indications, limits and non indications. Interventional Neuroradiologic, 14, 60-62. 